\title{
Panorama
}

8

Meldungen aus Praxis und Forschung

\section{Im Blickpunkt}

13 Interessenkonflikte in der Medizin

Wie unabhängig können Ärzte sein?

\section{Literatur kompakt}

17

20

22 Tumoren der Lunge

29

\section{Prädiktive Biomarker beim Rektumkarzinom \\ Kim JW et al. Int J Radiat Oncol Biol Phys. 2012;84(5):e577-84. \\ kommentiert von Erhard Hiller, München}

35 Lymphödem-Risiko nach SLNB gering

McLaughlin SA et al. J Am Coll Surg. 2013;216(3):380-9.

kommentiert von Wolfram Malter, Köln

42

Urogenitale Tumoren

46 Supportivtherapie

47 Allgemeine Onkologie

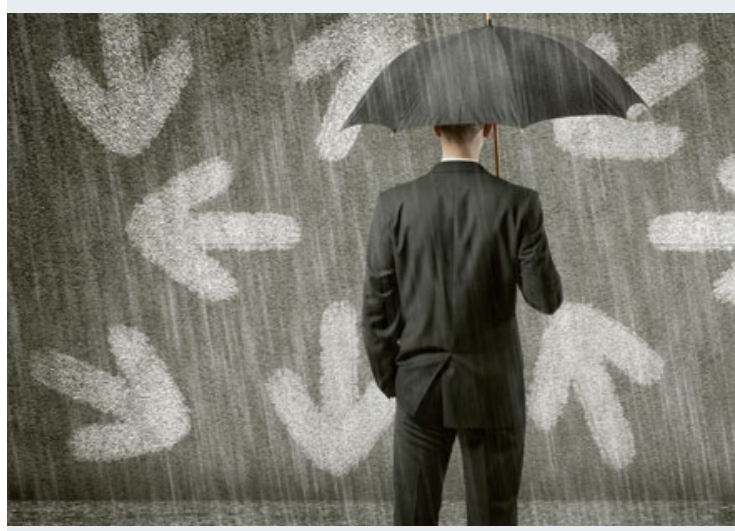

13 Medizinische Unabhängigkeit Mit Interessenkonflikten aller Art sind Ärzte beinahe täglich konfrontiert. Kritiker wollen die Mediziner von rein ökonomisch gesteuerten Zielvorgaben befreien und den Einfluss der Pharmaindustrie auf ärztliche Entscheidungen eingrenzen.

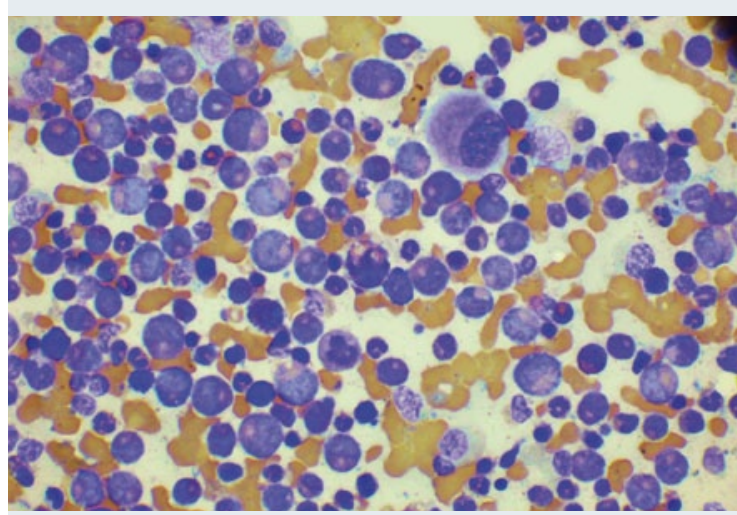

21 Tyrosinkinaseinhibition bei CML Der Tyrosinkinaseinhibitor Imatinib markierte einen Meilenstein in der Behandlung der chronischen myeloischen Leukämie. Nun wurde untersucht, ob Patienten mit Unverträglichkeit oder Resistenz gegen Imatinib von einem TKI der zweiten Generation profitieren.

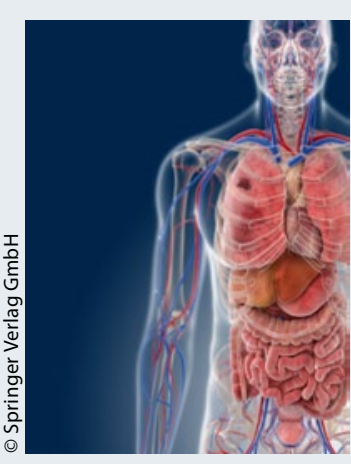

Titelbild \section{.}

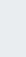
.

.

.

\section{Verlagsredaktion \\ Doris Berger (Leitung) \\ E-Mail: doris.berger@springer.com \\ Kim Jené (Redaktion) \\ E-Mail: kim.jene@springer.com}

Verlag Urban \& Vogel GmbH

Aschauer Str. 30

81549 München

Besuchen Sie uns online: www.springermedizin.de/ im-focus-onkologie

\section{springermedizin.de auf Twitter}

Das Zwitschern wird immer lauter: Werden Sie zum "Follower" und "lauschen" Sie unseren Springer-Medizin-Tweets auf www.twitter.com - oder mit dem Twitter-App auf Ihrem Smartphone.

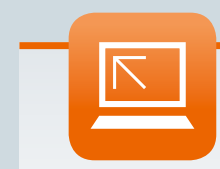

springermedizin.de aufTwitter

http://www.springermedizin.de 


\section{Fortbildung}

\section{ASORS - Supportivtherapie und Rehabilitation}

51 Kompetenter Umgang gefordert Komplementärmedizin in der Onkologie Markus Horneber und Matthias Rostock

\section{CME - Zertifizierte Fortbildung}

56 Nebennierentumoren

Diagnostische Odyssee

Natalie Wagner und Dorothea Weckermann

\section{Medizin aktuell}

\section{ASCO GI 2013: Lebermetastasen im Fokus}

SIRT zeigt vielversprechenden klinisch relevanten Nutzen

$64 \quad 2^{\text {nd }}$ World Congress of Cutaneous Lymphomas 2013

Gezielte Therapien für seltene Lymphome

66

DGHO-Frühjahrstagung

Wege aus der Zytostatikaklemme

\section{Praxis konkret}

\section{Zukunft der Onkologie}

69 Ambulante spezialfachärztliche Versorgung

Spielregeln stehen fest

Wirtschaft + Recht

70 Qualitätsmanagement in Praxen

Nachzügler gibt es unter Vertragsärzten kaum mehr

\section{Menschen, Ideen, Perspektiven}

\section{Wer ist Wolfgang Dörr?}

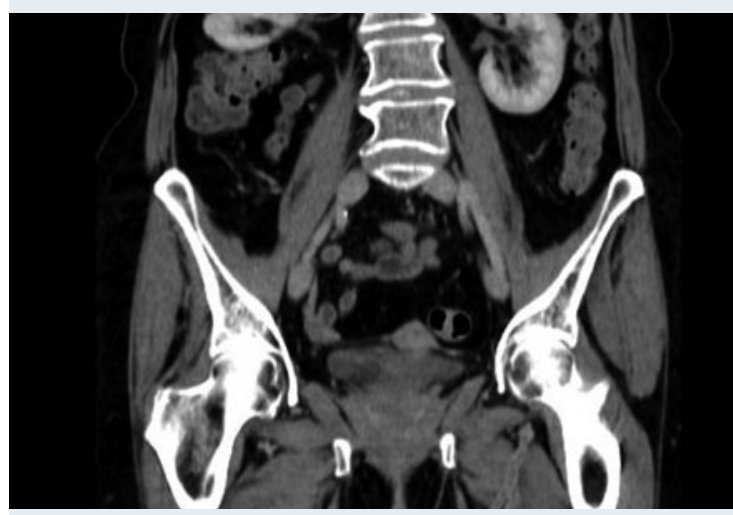

56 Tumoren der Nebennieren Der Weg bis zur Diagnose des sehr seltenen Nebennierenkarzinoms ist für Patient und Arzt häufig lang und aufwendig. Diagnostik und Therapie sollten entsprechend der aktuellen Erkenntnisse erfolgen.

\section{Kutane Lymphome}

Bislang fehlen Daten zu zielgerichteten Therapien gegen kutane Lymphome. Aber auf dem Weltkongress für kutane Lymphome wurde deutlich, dass die Forschungsaktivitäten zunehmen.

\section{Bitte vormerken!}

\section{MASCC/ISOO 2013 in Berlin}

"The International Symposium on Supported Care in Cancer" der Multinational Association of Supportive Care in Cancer (MASCC) und der International Society of Oral Oncology (ISOO) wird vom 27. bis 29. Juni 2013 in Berlin stattfinden.

Programm und weitere Informationen: http://www2.kenes.com/mascc2013

\section{Rubriken}

48 Quiz

61 CME-Fragebogen

72 Pharmaforum

76 Impressum 